\title{
BMJ Open Frailty and driving status associated with disability: a 24-month follow-up longitudinal study
}

Takehiko Doi (D) , Kota Tsutsumimoto, Hideaki Ishii, Sho Nakakubo, Satoshi Kurita, Hiroyuki Shimada

To cite: Doi T, Tsutsumimoto K, Ishii $\mathrm{H}$, et al. Frailty and driving status associated with disability: a 24-month follow-up longitudinal study. BMJ Open 2021;11:e042468. doi:10.1136/ bmjopen-2020-042468

- Prepublication history for this paper is available online. To view these files, please visit the journal online (http://dx.doi. org/10.1136/bmjopen-2020042468).

Received 06 July 2020 Revised 19 January 2021 Accepted 31 March 2021

\section{ABSTRACT}

Objectives To examine the relationship of driving status and frailty with disability in older adults.

Design A prospective study.

Setting and participants The study included 8533 participants (mean age: $72.0 \pm 6.1$ years (range: $60-98$ years), women: $54.1 \%$ ) in a community setting. Measures Driving status and frailty were assessed at baseline. The clinical definition of frailty was used according to the Japanese Cardiovascular Health Study index. Disability was prospectively determined using a record of Japanese long-term care insurance (LTCl). Results During the follow-up period (mean duration: 23.5 months), 58 (0.7\%) participants were regarded as moving out of the city, $80(0.9 \%)$ participants had died and 311 (3.6\%) participants were certified by LTCI. The proportion of disability was $1.3 \%$ among the not-frail group and $5.3 \%$ among the frail group. The proportion of disability was $2.5 \%$ in participants who were currently driving and $7.5 \%$ in those not driving. Based on frailty status and driving, participants were further classified into four groups: not frail and currently driving $(n=2945)$, not frail and not driving ( $n=642)$, frail and currently driving $(n=3598)$ and frail and not driving $(n=1348)$. Compared with older adults who are not frail and driving, the combined status of frail and not driving (adjusted HR: 2.28; 95\% Cl: 1.47 to 3.52) and frail and driving (HR: 1.91; 95\% Cl: 1.30-2.81) were risk factors for disability.

Conclusions Not driving and frail were associated with a risk of disability in community-dwelling older adults.

\section{INTRODUCTION}

Extending healthy life expectancy and diminishing the duration of life with disability help to decrease health burdens on society. ${ }^{1}$ Frailty is regarded as a prodromal stage of disability and has a high risk of adverse health outcomes, including disability. Frailty is a reversible status; someone's health could become robust again. ${ }^{2}$ Although frailty is a complex age-related clinical condition, adequate assessment of risk and preventive actions could help providers disrupt the progression from frail to disabled. ${ }^{3}$

Driving is a critical resource in supporting an active lifestyle in older adults. In fact,

\section{Strengths and limitations of this study}

- This is a large population study including over 8000 older adults.

- Frailty was defined by the Japanese CHS index.

- Incident disability was followed over time using data from long-term care insurance.

- The primary limitation was the short follow-up duration.

- Baseline data were collected from health checkups that had a selection bias.

driving cessation increases the risk of disability. ${ }^{45}$ However, whether the combined condition of not driving and being frail also elevates the risk of disability is unclear. More older adults are driving cars, but they are also having more accidents, especially in super-aged societies like Japan. ${ }^{6}$ Adequate evaluation of driving ability is required, but driving cessation with insufficient cause can potentially increase disability. Thus, our study aimed to elucidate how frailty and driving status affect disability risks.

\section{METHODS}

Participants

Participants in this study were from the National Center for Geriatrics and Gerontology-Study of Geriatric Syndromes, ${ }^{7}$ which aims to establish a screening system for geriatric syndromes and validate evidencebased interventions for preventing these syndromes. Participants were collected from surveys conducted in 2015-2017; 9701 individuals aged 60 years or over were eligible. The survey was regarded as the baseline and prospectively collected data were used for a follow-up duration of approximately 2 years (23.5 months). Data to be certified with long-term care insurance (LTCI) were collected during the follow-up period. Other variables including frailty, driving status and 
covariates were assessed at baseline. Exclusion criteria included diagnoses of dementia, stroke and Parkinson's disease; being unable to independently perform basic activities of daily living; being certified with LTCI in Japan before the survey; and having missing values for analysis. All participants provided written informed consent.

\section{Frailty}

The definition of frailty used in this study was the Japanese CHS (J-CHS) index ${ }^{8-10}$ according to CHS index criteria. ${ }^{2}$ The components of frailty in the J-CHS index are the same as those in the original CHS index: shrinking (weight loss), weakness, poor endurance (exhaustion), slowness and low activity. Weight loss was collected by a question from the Kihon Checklist ${ }^{11}$ with the question 'Have you lost $2 \mathrm{~kg}$ or more in the past 6 months?' A 'yes' answer indicated that participants had experienced weight loss. The Kihon Checklist is a self-administered questionnaire to identify frail older adults who are at risk of being newly certified for LTCI in the near future consisting of 25 items in the following categories: physical strength, nutritional status, oral function, cognitive function, houseboundness and depression risk. ${ }^{11}$ Weakness was defined as low muscle strength based on grip strength, measured using a Smedley-type handheld dynamometer (Takei, Niigata, Japan). Sex-specific cut-offs of low muscle strength were $<26 \mathrm{~kg}$ in men and $<18 \mathrm{~kg}$ in women. ${ }^{89} 12$ Exhaustion was assessed using a question from the Kihon Checklist ${ }^{11}$ : a 'yes' answer to the question 'In the last 2 weeks, have you felt tired for no reason?' indicated that participants had exhaustion or poor endurance. Slowness was defined as slow walking speed under normal conditions. Participants were asked to walk on a straight $6.4 \mathrm{~m}$ walkway on a flat floor with their usual gait speed. Gait time was measured over a $2.4 \mathrm{~m}$ distance between marks at $2.0 \mathrm{~m}$ and $4.4 \mathrm{~m}$ from the start of the walkway, and the mean gait speed $(\mathrm{m} / \mathrm{s})$ was calculated. The cut-off value of slowness was $<1.0 \mathrm{~m} / \mathrm{s} .{ }^{89}$ Low activity level was also measured using the questionnaire and indicated through a response of 'no' to both: 'Do you engage in moderate levels of physical exercise or sports aimed at health?' and 'Do you engage in low levels of physical exercise aimed at health? ${ }^{89}$ Based on the values of these five components (weight loss, weakness, exhaustion, slowness and low activity), our study assigned 'frail' to values of 1 and over, including prefrailty (1-2) and frailty (3 or over).$^{89}$

\section{Driving status}

The survey asked participants about their driving status. To determine driving status, current status of driving license (without license (never having a license), surrendered license, license not renewed, has license but not driving and currently driving with license) was reviewed. In our study, the status of currently driving with license was regarded as currently driving, and all other statuses were regarded as not driving.

\section{Disability}

LTCI certification in all participants was monitored throughout the follow-up period. LTCI certifies a person as 'Support Level 1 or 2' if he or she needs support for daily activities or 'Care Level 1, 2, 3, 4 or 5' if they need continuous care. ${ }^{13}$ Beneficiaries of the LTCI can use multiple services for which they are eligible. They can use more services than are covered if they pay all the costs for services beyond the maximum level. ${ }^{13} 14$ In our study, becoming disabled was defined as a new LTCI certification at any level. If we were unable to follow-up and assess for incident disability (due to moving out of the city and death), this was treated as censored data. We monitored this information through monthly updates. We defined the follow-up period as beginning at the time we conducted the survey at baseline (mean follow-up duration: 23.5 months (max: 24.0 months)).

\section{Covariates}

To understand participants' characteristics, demographic data, medical condition and lifestyle were assessed. Regarding demographic data, age and sex were collected. For medical condition, participants were interviewed about their medication use by well-trained nurses or other medical staff and medication numbers were collected. Cognitive function was assessed through the Mini-Mental State Examination (MMSE).${ }^{15}$ Depressive symptoms were assessed using the Geriatric Depression Scale (15 items version). ${ }^{16}$ In addition, going out less frequently was assessed by a question from the Kihon Checklist. ${ }^{11}$

\section{Statistical analysis}

Variables at baseline were compared between participants with and without disability during the follow-up period using an unpaired t-test or $\chi^{2}$ test. Participants were classified into four groups according to their baseline status of driving and frailty: not frail and currently driving, not frail and not driving, frail and currently driving, and frail and not driving.

Variables at baseline were compared between participants with and without disability during the follow-up period using an unpaired t-test or $\chi^{2}$ test. Participants were classified into four groups according to their baseline status of driving and frailty: not frail and currently driving, not frail and not driving, frail and currently driving, and frail and not driving.

To examine the association of frailty and driving status with the risk of disability, Kaplan-Meier survival risk assessments were used to plot cumulative survival function, and the results for each group were compared using log-rank tests. The objective variable was set as incident disability, and the explanatory variable was four groups based on frailty status and driving. In addition, Cox proportional hazards regression models were used to test the association. For incident disability, HRs of the frail group compared with the not-frail group and the not-driving 


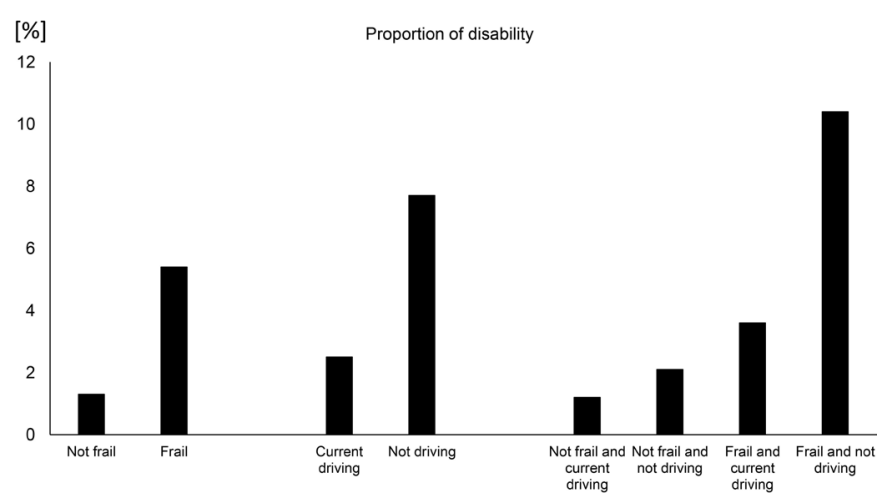

Figure 1 Proportion of disability between frailty and driving statuses. The definition of frail for classification into groups was prefrailty or frailty.

group compared with the driving group were set respectively between models as well as in the same model. Four groups were also set as the explanatory variable, with the not-frail and currently driving group as reference. These analyses were conducted in crude and adjusted models, including covariates. In addition, for a sensitivity analysis, Cox proportional hazards regression analysis was also conducted to establish a different definition of disability. In the sensitivity analysis, disability was defined to be certified as 'Care Level 1 or higher,' and other variables were set in the same manner. Each model in the Cox proportional hazards regression analysis calculated HR and $95 \%$ CIs. All analyses were performed using SPSS statistics software, V.20 (IBM). Statistical significance was set at $\mathrm{p}<0.05$ in all analyses.

Table 1 Characteristics of participants with disability and without during the follow-up period

\begin{tabular}{|c|c|c|c|}
\hline Variables & $\begin{array}{l}\text { Without } \\
\text { disability } \\
(n=8084)\end{array}$ & $\begin{array}{l}\text { With } \\
\text { disability } \\
(n=311)\end{array}$ & $P$ value \\
\hline Age, years & $71.7(5.9)$ & $79.2(6.3)$ & $<0.001$ \\
\hline Sex (women), \% & 54.1 & 56.3 & 0.450 \\
\hline Medication numbers & $2.7(2.6)$ & $3.8(2.8)$ & $<0.001$ \\
\hline $\begin{array}{l}\text { Less frequent going } \\
\text { out, \% }\end{array}$ & 13.2 & 33.5 & $<0.001$ \\
\hline Currently driving, \% & 77.8 & 51.8 & $<0.001$ \\
\hline Status of frailty & & & $<0.001$ \\
\hline Robust, \% & 43.2 & 15.1 & \\
\hline Prefrailty, \% & 50.2 & 52.4 & \\
\hline Frailty, \% & 6.6 & 32.5 & \\
\hline $\begin{array}{l}\text { Geriatric depression } \\
\text { score }\end{array}$ & $2.6(2.5)$ & $3.8(2.7)$ & $<0.001$ \\
\hline MMSE, score & $27.3(2.4)$ & $25.0(3.6)$ & $<0.001$ \\
\hline
\end{tabular}

Values are means (SD) or proportions. The total number of participants were 8533. Data in this table excluded 138 participants (58 participants were regarded as moving out and 80 participants had died).

MMSE, Mini-Mental State Examination.
Patient and public involvement

This study was conducted without patient or public involvement.

\section{RESULTS}

From 9701 eligible participants, 8533 (mean age: $72.0 \pm 6.1$ years (age range: 60-98 years), women: $54.1 \%$ ) participants were matched with criteria and analysed in this study. During the follow-up period, $58(0.7 \%)$ participants were regarded as moving out, $80(0.9 \%)$ participants died and 311 (3.6\%) participants were certified by LTCI. Participants with disability, compared with those without disability, were older, took more medications, went out less frequently and had higher scores on the Geriatric Depression Scale and lower scores on MMSE (table 1, all $\mathrm{p}<0.001)$. This comparison used data excluded 138 participants (58 participants were regarded as moving out and 80 participants had died). In addition, participants with disabilities were more likely to be frail and less likely to be currently driving (both $\mathrm{p}<0.001$ ).

The proportion of incident disability during the follow-up period was dependent on the status of frailty and driving (figure 1). The proportion of participants with disability was $1.3 \%$ in the not-frail group and $5.3 \%$ in the frail group. The proportion of participants with disability who were currently driving was $2.5 \%$, and the proportion of participants who were not driving was $7.5 \%$. Based on their status of frailty and driving, participants were further classified into four groups: not frail and currently driving $(\mathrm{n}=2945)$, not frail and not driving $(\mathrm{n}=642)$, frail and currently driving $(\mathrm{n}=3598)$ and frail and not driving $(n=1348)$. Among those four groups, participants who were not frail and currently driving had the lowest proportion of disability $(1.2 \%)$ and participants who were frail and not driving had the highest proportion of disability $(10.2 \%)$. Survival risk based on log-rank test did not show a difference between participants from the not-frail and currently driving group and the not-frail and not-driving group $(\mathrm{p}=0.077)$. There were also no significant differences between participants from the not-frail and not-driving group and from the frail and currently driving group $(\mathrm{p}=0.051)$. Other intergroup results did show differences $(p<0.001)$. From the analysis using Cox proportional hazards regression models, the frail group had an increased risk of disability (crude HR: 4.15 [95\% CI: 3.04 to 5.66), as did not driving (crude HR: 3.15 ; $95 \%$ CI: 2.52 to 3.93 ). In the model that set frailty and driving status together, similar results were shown for frailty (HR: 3.72; 95\% CI: 2.72 to 5.07 ) and not driving (HR: 2.79; 95\% CI: 2.23 to 3.49). In addition, compared with the not-frail and currently driving group, participants from the frail and currently driving group $(p=0.001)$, and those from the frail and not-driving group $(\mathrm{p}<0.001)$, had an increased risk of incident disability (table 2). For a sensitivity analysis, a different definition of disability (being certified as 'Care Level 1 or higher') was used. Adjusted HR for incident disability was higher 
Table 2 Association of status in frailty and driving with disability

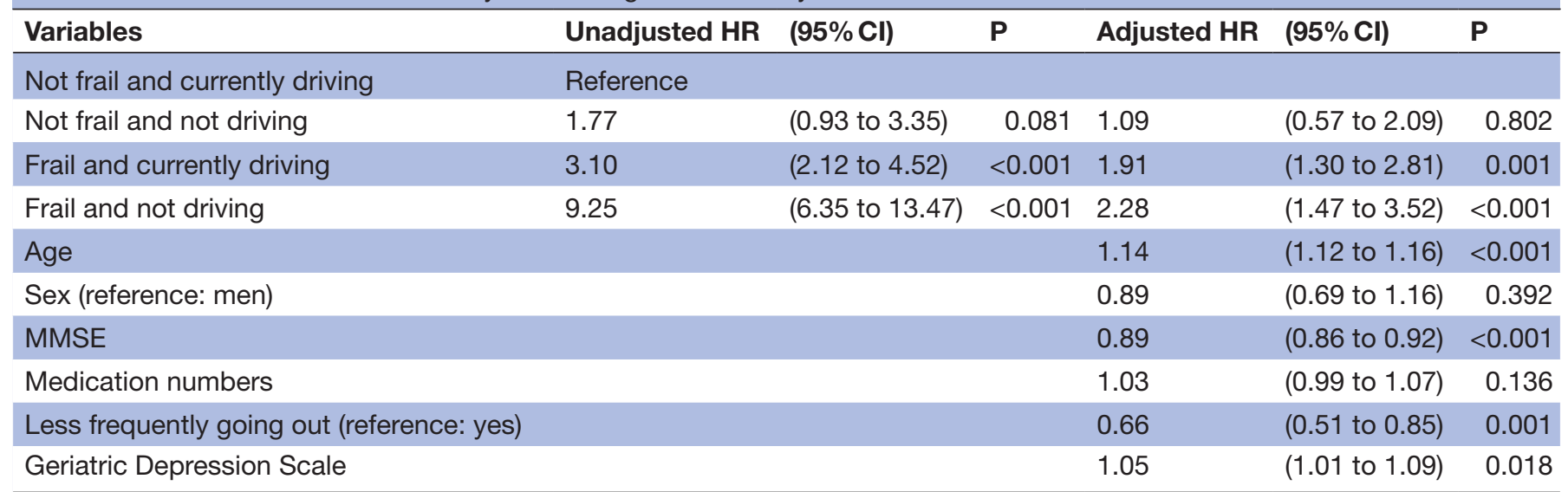

The total number of participants was 8533 . The definition of frail for group classification was prefrailty or frailty. MMSE, Mini-Mental State Examination.

in the frail and not-driving group (HR: $1.87 ; 95 \%$ CI: 1.06 to 3.31), and other groups (not frail and not driving; frail and currently driving) were not significantly associated with disability.

\section{DISCUSSION}

Our study examined the association of frailty and driving status with the risk of disability among older adults. The proportion of incident disability was higher among those with a status of frail and not driving. The effects of driving were observed among participants who were frail, while not driving did not increase the risk of disability among participants who were not frail. This result remained even after adjustment with covariates.

Our study revealed that being frail was associated with a risk of incident disability, which is in line with previous research. Numerous studies have indicated that frailty caused disability and other adverse health outcomes. ${ }^{2}$ When we studied data from another section of the National Center for Geriatrics and Gerontology - Study of Geriatric Syndromes: NCGG-SGS in 2011-2012 with a similar follow-up duration (about 2 years), we saw a similar risk for frailty to cause disability, ${ }^{10}$ although the data in 2011-2012 did not have detailed assessments regarding driving. Our study is in accordance with similar studies and expands the previous evidence regarding frailty. Driving status had been associated with a risk of disability, particularly among frail participants. Driving a motor vehicle has a beneficial role in maintaining life space and activities in older adults. In fact, having a valid driving license was associated with reduced hazard of life-space constriction ${ }^{17}$ and not driving increased the restriction of life-space restriction. ${ }^{18}$ In addition, having a combined status of frail and not driving created a high risk of disability compared with the other statuses. Not driving also caused functional decline in older adults, particularly when driving ceased. A prospective study revealed that stopping driving or reducing the distance driven was related to several functional declines and a decline in instrumental activities of daily living. ${ }^{19}$ Furthermore, not driving was associated with a higher risk of mortality. ${ }^{20} 21$ O'Connor suggested that the relationship may be explained by health difficulties in social, physical and general health to accompany or follow driving cessation. ${ }^{20}$

Our results brought to light new ideas about the assessment of driving continuity in older adults. By engaging in several activities associated with driving, older adults may successfully age. The current system in Japan for adults aged 70 years or over, established by Japan's National Police Agency, requires individuals to attend a lecture on driving operation, undergo vision tests, attend an on-road lecture and be screened for cognitive function. ${ }^{22}$ If they are appropriately diagnosed as having dementia, they are unable to renew their licenses. ${ }^{22}$ The evaluation of physical function (ie, frailty) should be considered as part of the assessments used to renew licenses. Furthermore, age-related changes that affect driving skill are varied and occurred gradually. Thus, offering restricted licenses (eg, restricting legal driving times to daylight or good weather) should be considered before cessation. To introduce such limited licenses may require detailed assessments in addition to the current system.

The strength of this study was that it was conducted in a large population study with a prospective design. Our study also has some limitations. In this study, disability was defined as certification of LTCI. LTCI cannot systematically distinguish causes for the disability certification. For example, we could not objectively differentiate between mobility and cognitive disabilities. In addition, LTCI has several levels (Support Level 1-2, Care Level 1-5) based on the results of standardised assessments and a final decision from the expert board (Nursing Care Needs Certification Board). Characteristics of participants with disabilities are thus varied and depend on certified levels. Further study including more participants is required to compare the differences of each certified level. Furthermore, the data used in our study were derived from the NCGG-SGS database based on invitational health checkups among Japanese older adults. Such 
check-ups have a selection bias in that participants may have a higher health literacy. Therefore, the results of our study are not easily generalisable. In addition, data in this study could not clarify casual association between driving and disability. Reverse causation (that functional decline with disability affects driving cessation) is also possible. To examine reverse causation, data regarding changes in function, incident disability and future driving cessation should be analysed. Next, our study used the J-CHS index to define frailty; using other criteria to define frailty may affect the results. Finally, detailed driving statuses (eg, frequency, driving under specific conditions such as at night and during bad weather) could not be considered in the analysis in this study due to limitations in the data. Further studies with sufficient cohort data and intervention studies should be conducted in the future.

In conclusion, frailty and driving status were found to be associated with the risk of disability. Not driving increased the risk of disability, particularly among frail older adults. The status of driving should be considered to assess the risk of disability.

\section{Acknowledgements We thank the Obu City and Takahama City Office for help} with participant recruitment.

Contributors TD was responsible for acquisition of the data, statistical analysis, interpretation of the data and drafting of the manuscript. HS helped in study design and concept, and drafting and revising of the manuscript. HI and KT were responsible for acquisition of the data, statistical analysis, interpretation of the data and drafting of the manuscript. SN and SK helped in acquisition and interpretation of the data and drafting of the manuscript.

Funding This work was supported by AMED under grant number (15dk0207004h0203, 15dk0107003h0003); a Grant-in-Aid for Scientific Research (B) (grant number 23300205); the Funds of Obu City Local Government; and Research Funding for Longevity Sciences $(26-33,29-31,30-7)$ from the National Center for Geriatrics and Gerontology, Japan.

Competing interests None declared.

Patient and public involvement Patients and/or the public were not involved in the design, or conduct, or reporting, or dissemination plans of this research.

Patient consent for publication Not required.

Ethics approval The ethics committee of the National Center for Geriatrics and Gerontology approved this study $(770,791)$.

Provenance and peer review Not commissioned; externally peer reviewed.

Data availability statement № data are available.

Open access This is an open access article distributed in accordance with the Creative Commons Attribution Non Commercial (CC BY-NC 4.0) license, which permits others to distribute, remix, adapt, build upon this work non-commercially, and license their derivative works on different terms, provided the original work is properly cited, appropriate credit is given, any changes made indicated, and the use is non-commercial. See: http://creativecommons.org/licenses/by-nc/4.0/.

\section{ORCID iDs}

Takehiko Doi http://orcid.org/0000-0003-0205-8663

Hiroyuki Shimada http://orcid.org/0000-0001-8111-6440

\section{REFERENCES}

1 GBD 2017 DALYs and HALE Collaborators. Global, regional, and national disability-adjusted life-years (DALYs) for 359 diseases and injuries and healthy life expectancy (HALE) for 195 countries and territories, 1990-2017: a systematic analysis for the global burden of disease study 2017. Lancet 2018;392:1859-922.

2 Fried LP, Tangen CM, Walston J, et al. Frailty in older adults: evidence for a phenotype. J Gerontol A Biol Sci Med Sci 2001;56:M146-57.

3 Hoogendijk EO, Afilalo J, Ensrud KE, et al. Frailty: implications for clinical practice and public health. Lancet 2019;394:1365-75.

4 Hirai $\mathrm{H}$, Ichikawa $\mathrm{M}$, Kondo $\mathrm{N}$, et al. The risk of functional limitations after driving cessation among older Japanese adults: the JAGES cohort study. J Epidemiol 2020;30:332-7.

5 Shimada H, Makizako H, Tsutsumimoto K, et al. Driving and incidence of functional limitation in older people: a prospective population-based study. Gerontology 2016;62:636-43.

6 National Police Agency. The white paper on police, 2019.

7 Shimada H, Tsutsumimoto K, Lee S, et al. Driving continuity in cognitively impaired older drivers. Geriatr Gerontol Int 2016;16:508-14.

8 Satake S, Shimada H, Yamada M, et al. Prevalence of frailty among community-dwellers and outpatients in Japan as defined by the Japanese version of the cardiovascular health study criteria. Geriatr Gerontol Int 2017;17:2629-34.

9 Shimada H, Makizako H, Doi T, et al. Incidence of disability in frail older persons with or without slow walking speed. J Am Med Dir Assoc 2015;16:690-6.

10 Makizako H, Shimada H, Doi T, et al. Impact of physical frailty on disability in community-dwelling older adults: a prospective cohort study. BMJ Open 2015;5:e008462.

11 Fukutomi E, Okumiya K, Wada T, et al. Relationships between each category of 25 -item frailty risk assessment (Kihon checklist) and newly certified older adults under long-term care insurance: a 24-month follow-up study in a rural community in Japan. Geriatr Gerontol Int 2015;15:864-71.

12 Chen L-K, Liu L-K, Woo J, et al. Sarcopenia in Asia: consensus report of the Asian Working group for sarcopenia. J Am Med Dir Assoc 2014;15:95-101.

13 Tsutsui T, Muramatsu N. Japan's universal long-term care system reform of 2005: containing costs and realizing a vision. J Am Geriatr Soc 2007; 55:1458-63.

14 Tsutsui T, Muramatsu N. Care-needs certification in the long-term care insurance system of Japan. J Am Geriatr Soc 2005;53:522-7.

15 Folstein MF, Folstein SE, McHugh PR. "Mini-mental state." A practical method for grading the cognitive state of patients for the clinician. J Psychiatr Res 1975;12:189-98.

16 Yesavage JA. Geriatric depression scale. Psychopharmacol Bull 1988;24:709-11.

17 Shah RC, Maitra K, Barnes LL, et al. Relation of driving status to incident life space constriction in community-dwelling older persons: a prospective cohort study. J Gerontol A Biol Sci Med Sci 2012;67:984-9.

18 Tsuji T, Rantakokko M, Portegijs E, et al. The effect of body mass index, lower extremity performance, and use of a private CAR on incident life-space restriction: a two-year follow-up study. BMC Geriatr 2018;18:271.

19 Marie Dit Asse L, Fabrigoule C, Helmer C, et al. Automobile driving in older adults: factors affecting driving restriction in men and women. J Am Geriatr Soc 2014;62:2071-8.

20 O'Connor ML, Edwards JD, Waters MP, et al. Mediators of the association between driving cessation and mortality among older adults. J Aging Health 2013;25:249S-69.

21 Edwards JD, Perkins M, Ross LA, et al. Driving status and three-year mortality among community-dwelling older adults. J Gerontol A Biol Sci Med Sci 2009;64:300-5.

22 Cabinet Office. White paper on traffic safety in Japan, 2016. 